\title{
Controllable microfluidic production of multicomponent multiple emulsions $\dagger$
}

\author{
Wei Wang, ${ }^{a}$ Rui Xie, ${ }^{* a}$ Xiao-Jie Ju, ${ }^{a}$ Tao Luo, ${ }^{a}$ Li Liu, ${ }^{a}$ David A. Weitz ${ }^{b}$ and Liang-Yin Chu ${ }^{* a}$ \\ Received 23rd January 2011, Accepted 9th March 2011 \\ DOI: 10.1039/c1lc20065h
}

\begin{abstract}
A hierarchical and scalable microfluidic device constructed from a combination of three building blocks enables highly controlled generation of multicomponent multiple emulsions. The number, ratio and size of droplets, each with distinct contents being independently co-encapsulated in the same level, can be precisely controlled. The building blocks are a drop maker, a connector and a liquid extractor; combinations of these enable the scale-up of the device to create higher-order multicomponent multiple emulsions with exceptionally diverse structures. These multicomponent multiple emulsions offer a versatile and promising platform for precise encapsulation of incompatible actives or chemicals, for synergistic delivery and biochemical and chemical reactions, and for engineering multicompartment materials with controlled internal phases.
\end{abstract}

Multiple emulsions are complex nested liquid systems, with liquid droplets of decreasing sizes placed one inside another. They are widely used as templates for the synthesis of multicompartment microspheres ${ }^{1-5}$ and core-shell microcapsules, ${ }^{6-10}$ as microreactors for chemical reactions, ${ }^{11}$ and as encapsulants for actives such as drugs $^{12-16}$ and nutrients. ${ }^{17-19}$ Multiple emulsions containing droplets with different contents in the same internal level are important for co-encapsulating incompatible actives or chemicals for synergistic delivery or for biochemical or chemical reactions, and for fabricating hierarchical multicompartment materials. ${ }^{20,21}$ Multiple emulsions are often generated through a multistep bulk emulsification process by sequential mixing, but these are exceedingly polydisperse. $^{22}$ Membrane emulsification techniques can produce monodisperse multiple emulsions, but the number of inner droplets is poorly controlled. ${ }^{15}$ Microfluidic techniques are an excellent method for manipulation of emulsions, ${ }^{23-30}$ both glass capillary devices $^{23,24}$ and poly(dimethylsiloxane) devices ${ }^{25-28}$ enable precise preparation of monodisperse multiple emulsions, with the size and number of droplets at each level in multiply nested emulsions being

${ }^{a}$ School of Chemical Engineering, Sichuan University, Chengdu, China. E-mail: chuly@scu.edu.cn; Tel: +8602885460682; xierui@scu.edu.cn ${ }^{b}$ School of Engineering and Applied Sciences/Department of Physics, Harvard University, Cambridge, USA

$\dagger$ Electronic supplementary information (ESI) available: S1: Experimental details; S2: results and discussion: the number, ratio and size control of inner different droplets; the independent size control of outer droplets by connector and liquid extractor; and the development of prediction equations. See DOI: 10.1039/c1lc20065h precisely controlled. However, the control of the number, ratio and size of different droplets within each level, and the co-encapsulation of multicomponent droplets for higher-order multiple emulsions, cannot be easily achieved with these devices. Controlled, higherorder, multicomponent multiple emulsions would offer advanced platforms for the design of more complex multicompartment materials, and would provide synergistic delivery systems or chemical microreactors for incompatible actives or chemicals with more precise encapsulation and regulated mass-transfer kinetics. Co-encapsulation of two droplets containing different contents in double emulsions has been accomplished with glass microfluidic devices using a cross-junction structure $\mathrm{e}^{31,32}$ and with capillary microfluidic devices with two separate internal channels in the injection tube. ${ }^{20}$ However, the precise control over the number, ratio and size of the co-encapsulated multicomponent droplets in double emulsions remains very difficult to achieve, and this is essential to fully exploit the power and potential of these structures. Thus, new techniques for controlled generation of higher-order multicomponent multiple emulsions are required.

Here we describe a hierarchical and scalable microfluidic device that generates multicomponent multiple emulsions where the number, ratio and size of the co-encapsulated droplets, containing different contents, are precisely controlled at each level. This novel microfluidic device consists of three basic building blocks, a drop maker, a connector and a liquid extractor; using different combinations enables a flexible and controllable method for generating varied higher-order multicomponent multiple emulsions. We demonstrate the scalability and controllability of the microfluidic device by starting with quadruple-component double emulsions, and scaling up by simply adding building blocks to generate quintuple-component double emulsions, quintuple-component triple emulsions, and even sextuple-component triple emulsions. In each case, the number, ratio and size of co-encapsulated multicomponent droplets are individually controlled with unprecedented accuracy.

The microfluidic device is comprised of three basic units, a drop maker, a connector and a liquid extractor (Fig. 1a, see the ESI $\dagger$ for details on the fabrication of the devices). The drop maker is designed for generating droplets (Fig. 1(a1)); the connector is designed for merging droplets from different drop makers (Fig. 1(a2)), and the liquid extractor is designed for removing unwanted fluid from the continuous phase (Fig. 1(a3)). By using different combinations of the building blocks we achieve a versatile route to creating microfluidic devices for the formation of multicomponent multiple emulsions with highly controlled yet exceptionally diverse structures. 
(a)

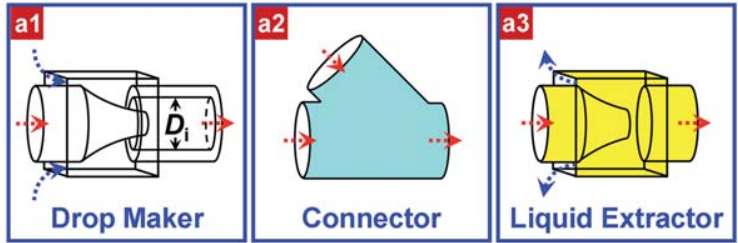

(b)

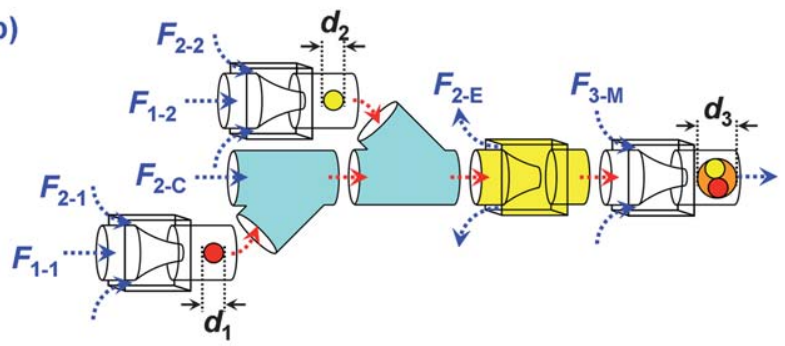

(c)

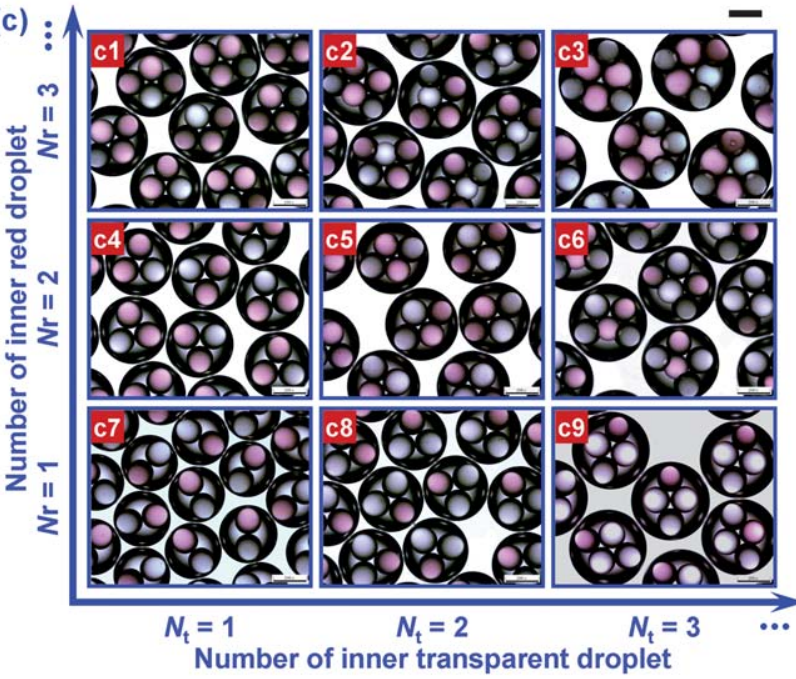

Fig. 1 Microfluidic device and controlled production of quadruplecomponent double emulsions. (a) Schematic diagram of functional building blocks: (a1) drop maker for generating droplets, (a2) connector for merging droplets from different drop makers, and (a3) liquid extractor for removing excess continuous phase. (b) Schematic diagram of the microfluidic device constructed from the building blocks for generating quadruple-component double emulsions, in which $d_{1}, d_{2}$ and $d_{3}$ are the diameters for inner red, inner transparent, and outer droplets respectively. $F_{i-j}$ is fluid phase for each level of multiple emulsions, where the subscript $i$ refers to the level number where $F_{i-j}$ exists in multiple emulsions by counting from the inside outward, and the subscript $j$ refers to the injection position of $F_{i-j}$ in the microfluidic device. The subscript $j$ can be the number or letter, where the number $(1,2, \ldots)$ means the number of branch channels, and letters $C, E$ and $M$ stand for connector, liquid extractor and main channel respectively. (c) Optical micrographs of quadruple-component double emulsions exhibiting the precise control over the number and ratio of inner red and transparent droplets. Scale bars are $200 \mu \mathrm{m}$.

We demonstrate the versatility and controllability of microfluidic devices based on these building blocks by first generating quadruplecomponent double emulsions, in each of which droplets with different contents can be co-encapsulated while precisely and independently controlling the number, ratio and size of the droplets. The microfluidic device comprises three drop makers, two connectors and one liquid extractor (Fig. 1b). First, droplets of two different inner fluids $\left(F_{1-1}\right.$ and $\left.F_{1-2}\right)$ are separately emulsified in drop makers in different branch channels. Then, through the connectors, these two sets of droplets flow into the main channel, where they are merged to form a linear array of droplets. Because of the periodic droplet formation in each drop maker, the two types of droplets are regularly and alternately aligned in the main channel. We control the spacing between the droplets by using a connector $\left(F_{2-\mathrm{C}}\right)$ to inject fluid and a separate extractor $\left(F_{2-E}\right)$ to remove the middle fluid as required. The middle fluid is further emulsified by the outermost fluid in the drop maker $\left(F_{3-\mathrm{M}}\right)$ downstream, forming double emulsions containing two different types of droplets. The controlled production of monodisperse quadruple-component double emulsions, made from oil-in-water-in-oil $(\mathrm{O} / \mathrm{W} / \mathrm{O})$, is shown in Fig. 1c. The number and ratio of inner red and transparent droplets are precisely manipulated (see the $\mathrm{ESI} \dagger$ for details of fluid components and flow rate conditions). Accurate regulation of the number and ratio is achieved by fine adjustment of the flow rates. We demonstrate the excellent controllability of our microfluidic device by independently changing the number of encapsulated inner red $\left(N_{\mathrm{r}}\right)$ and transparent $\left(N_{\mathrm{t}}\right)$ droplets; this also leads to precise control over the ratio $\left(R_{12}\right)$ of red droplets to transparent droplets (Fig. 1c). We illustrate this control by showing the resultant double emulsions as a plot of $N_{\mathrm{r}}$ as a function of $N_{\mathrm{t}}$, with each varying from 1 to 3 , in Fig. 1c. The independent control over $N_{\mathrm{r}}$ and $N_{\mathrm{t}}$ affords accurate manipulation of $R_{12}$ with desired values (see the ESI $\dagger$ for a detailed discussion on the flow-ratedependent control over the number and ratio of different co-encapsulated droplets in double emulsions). Because we use the dripping mechanism to provide highly control over the formation of droplets in our microfluidic device, the droplets are generated with good reproducibility and monodispersity. Usually, our experiments of emulsion generation are operated for at least $8 \mathrm{~h}$ every day, and the formation processes of emulsions always remain stable all the time during the experiments if no external disturbance happens.

We can also achieve excellent control over the droplet size $\left(d_{\mathrm{i}}\right)$ by changing the flow rates or the inner diameter $\left(D_{\mathrm{i}}\right)$ of the collection tube of drop makers. The size manipulation is illustrated by controllably generating quadruple-component double emulsions that contain smaller red droplets and larger blue droplets (see the $\mathrm{ESI} \uparrow$ for controlled generation of quadruple-component double emulsions containing two kinds of droplets with different sizes). These results demonstrate successful co-encapsulation of droplets with different contents in double emulsions, with the number, ratio and size of different inner droplets precisely and independently controlled. These co-encapsulated different droplets can be employed to separately load incompatible actives or chemicals, and can be employed as different compartments for multicompartment materials. The excellent control over the number, ratio and size of different inner droplets allows us to precisely and independently manipulate the encapsulation of each active or chemical and the internal structure of multicompartment materials. Furthermore, generation of quadruple-component double emulsions with inverse water-in-oil-in-water (W/O/W) type is also feasible with our device because its coaxial structure does not require modification of surface wettability for drop formation of different fluids.

The precise and independent control over the size of outer droplets can also be achieved using the functionality of the connectors and liquid extractors. Besides merging the main channel with branch channels, the connector is also designed to adjust the distance between droplets in the main channel by injecting additional fluid. When we increase the flow rate $\left(Q_{2-\mathrm{C}}\right)$ of the injecting 
fluid $\left(F_{2-C}\right)$ into the connector while keeping the other flow rates constant, the distance between droplets increases as does the size of the outer droplets $\left(d_{3}\right)$, due to the encapsulation of more fluid in the middle aqueous layer. This size control of outer droplets is shown in Fig. 2a. When the injecting flow rate ratio $Q_{2-C} d\left(Q_{1-1}+Q_{2-1}+\right.$ $\left.Q_{1-2}+Q_{2-2}-Q_{2-\mathrm{E}}\right)$ increases from $\sim 0.250$ to $\sim 1.333$, the volume ratio $V_{\mathrm{i}} / V_{0}$ of outer droplets increases from 1 to $\sim 1.683$. Importantly, in spite of the increase in $d_{3}$, the size of inner red droplets $\left(d_{1}\right)$ and transparent droplets $\left(d_{2}\right)$ remain unchanged, because the inner droplets are individually formed in branch channels. By contrast, when we increase the flow rate through the liquid extractor $\left(Q_{2-\mathrm{E}}\right)$ while keeping the other flow rates constant, the distance between droplets decreases and the outer droplets become smaller due to the removal of continuous fluid. Increasing the extracting flow rate ratio $Q_{2-\mathrm{E}} /\left(Q_{1-1}+Q_{2-1}+Q_{1-2}+Q_{2-2}+\right.$ $Q_{2-\mathrm{C}}$ ) from $\sim 0.034$ to $\sim 0.241$ results in a decrease of volume ratio $V_{\mathrm{i}} / V_{0}$ of outer droplets from 1 to $\sim 0.736$, with $d_{1}$ and $d_{2}$ unchanged (Fig. 2b). Because the data in Fig. 2 are measured by using several batches of emulsion samples, the error bars mean that there is a little difference between the droplet diameters from batch to batch. However, the dripping mechanism for droplet formation offers highly control of the droplets, so the emulsions are still highly monodisperse in each batch, i.e., the droplet formation is still wellcontrolled. These results illustrate the precise and independent size control over the outer droplets of a quadruple-component double emulsion (see the ESI $\uparrow$ for detailed discussion on the independent control over the size of outer droplets by connector and liquid extractor).

The excellent control over the number, ratio and size of coencapsulated droplets allows us to quantitatively predict the inner structure of quadruple-component double emulsions for these microfluidic devices. The total number of both inner red and transparent droplets $(N)$ encapsulated in double emulsions is controlled by matching the formation rates of inner red $\left(f_{1-1}\right)$ and transparent $\left(f_{1-2}\right)$ droplets with those of the outer droplets $\left(f_{3}\right)$. This flow-ratedependent formation rate can be regulated by adjusting the flow rates of the dispersed $\left(Q_{\mathrm{d}}\right)$ and continuous $\left(Q_{\mathrm{c}}\right)$ fluids in the drop maker module. ${ }^{24}$ For fixed device dimensions $\left(D_{\mathrm{i}}\right)$ and solution conditions, there is a linear dependence of $d_{\mathrm{i}} / D_{\mathrm{i}}$ on $Q_{\mathrm{d}} / Q_{\mathrm{c}}{ }^{24}$ Based on these relations, we can quantitatively predict the value of $N$ using eqn (1) (see the ESI $\dagger$ for detailed derivation of the equation):
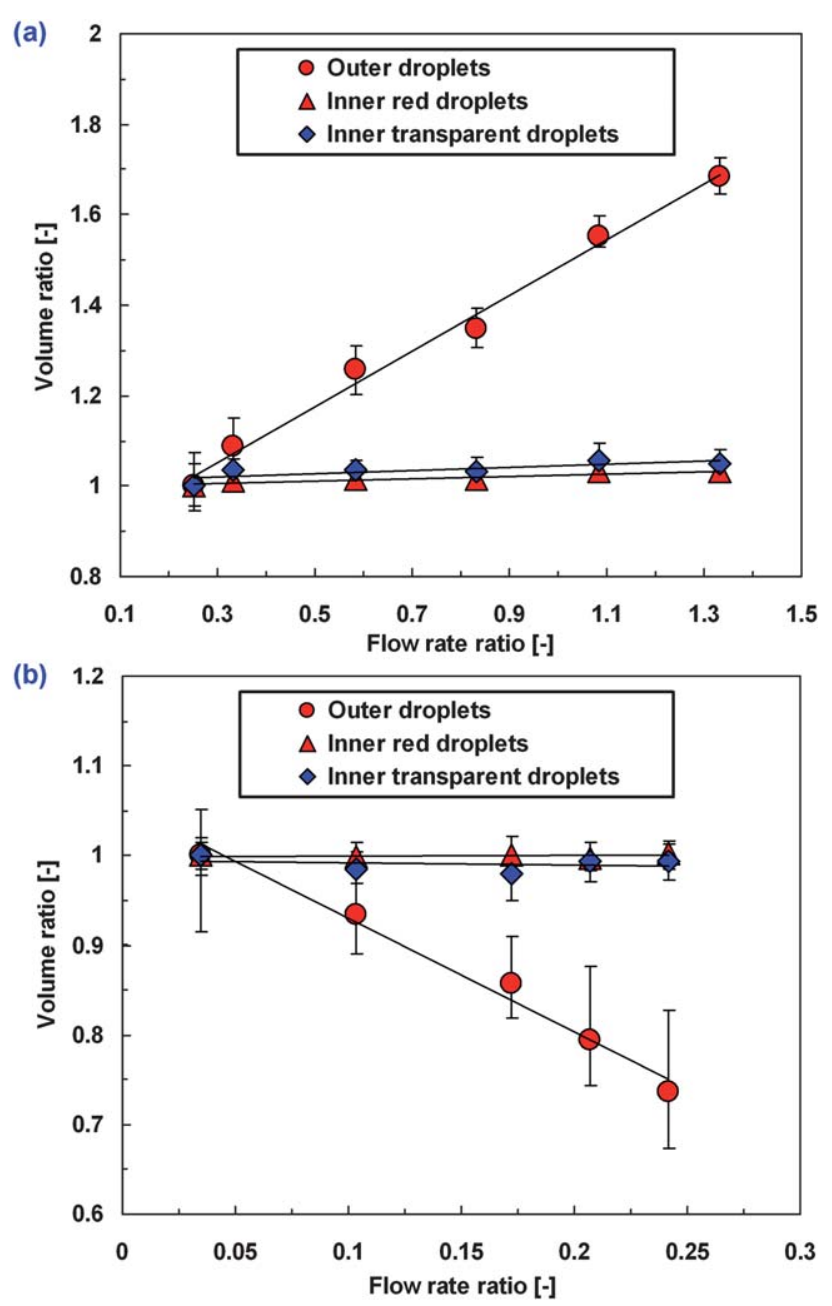

Fig. 2 Effects of the flow rates of connector (a) and liquid extractor (b) on the sizes of inner and outer droplets in quadruple-component double emulsions. (a) Droplet volume ratio $V_{\mathrm{i}} / V_{0}$ as a function of flow rate ratio $Q_{2-\mathrm{C}} /\left(Q_{1-1}+Q_{2-1}+Q_{1-2}+Q_{2-2}-Q_{2-\mathrm{E}}\right)$. The initial values of $d_{1}, d_{2}$ and $d_{3}$ are $163.2 \mu \mathrm{m}, 163.7 \mu \mathrm{m}$ and $353.1 \mu \mathrm{m}$, respectively. (b) Droplet volume ratio $V_{\mathrm{i}} / V_{0}$ as a function of flow rate ratio $Q_{2-\mathrm{E}} /\left(Q_{1-1}+Q_{2-1}+\right.$ $Q_{1-2}+Q_{2-2}+Q_{2-\mathrm{C}}$ ). $V_{0}$ is the initial droplet volume, and $V_{\mathrm{i}}$ is the droplet volume after varying $Q_{2-\mathrm{C}}$ (a) and $Q_{2-\mathrm{E}}(\mathrm{b})$. The initial values of $d_{1}, d_{2}$ and $d_{3}$ are $169.6 \mu \mathrm{m}, 168.5 \mu \mathrm{m}$ and $386 \mu \mathrm{m}$, respectively.

$$
N=\frac{f_{1-1}+f_{1-2}}{f_{3}}=\frac{\left(Q_{1-1} / D_{1}^{3}\left(a_{1}\left(Q_{1-1} / Q_{2-1}\right)+b_{1}\right)^{3}\right)+\left(Q_{1-2} / D_{2}^{3}\left(a_{2}\left(Q_{1-2} / Q_{2-2}\right)+b_{2}\right)^{3}\right)}{\left(Q_{3} / D_{3}^{3}\left(a_{3}\left(Q_{3} / Q_{3-\mathrm{M}}\right)+b_{3}\right)^{3}\right)}
$$

where $Q_{i-j}$ is the flow rate of fluid $F_{i-j} ; Q_{3}$ is the sum of $Q_{1-1}, Q_{2-1}$, $Q_{2-1}, Q_{2-2}, Q_{2-\mathrm{C}}$ and $Q_{2-\mathrm{E}}$ (Fig. 1b). Coefficients $a_{\mathrm{i}}$ and $b_{\mathrm{i}}$ are the slopes and intercepts that are obtained experimentally from the empirical relations for $d_{\mathrm{i}} / D_{\mathrm{i}}$ and $Q_{\mathrm{d}} / Q_{\mathrm{c}}$. The ratio of inner red droplets to transparent droplets $\left(R_{12}\right)$ depends on the number of each kind of droplet encapsulated in the double emulsion; this can be separately controlled by adjusting $f_{1-1}$ and $f_{1-2}$. Therefore, we can quantitatively determine the value of $R_{12}$ using eqn (2) (see the ESI $\dagger$ for detailed derivation of the equation):

$$
R_{12}=\frac{f_{1-1}}{f_{1-2}}=\frac{\left(Q_{1-1} /\left(\pi d_{1}^{3} / 6\right)\right)}{\left(Q_{1-2} /\left(\pi d_{2}^{3} / 6\right)\right)}=\frac{Q_{1-1}}{Q_{1-2}} \frac{D_{2}^{3}\left(a_{2}\left(Q_{1-2} / Q_{2-2}\right)+b_{2}\right)^{3}}{D_{1}^{3}\left(a_{1}\left(Q_{1-1} / Q_{2-1}\right)+b_{1}\right)^{3}}
$$

The calculated values of $N$ and $R_{12}$ from eqn (1) and (2) are compared with the experimental values in Fig. 3. The good agreement between the calculated and experimental values of both $N$ and $R_{12}$ indicates that we can successfully predict the inner structure of quadruple-component double emulsions. By exploiting the mass conservation of each 

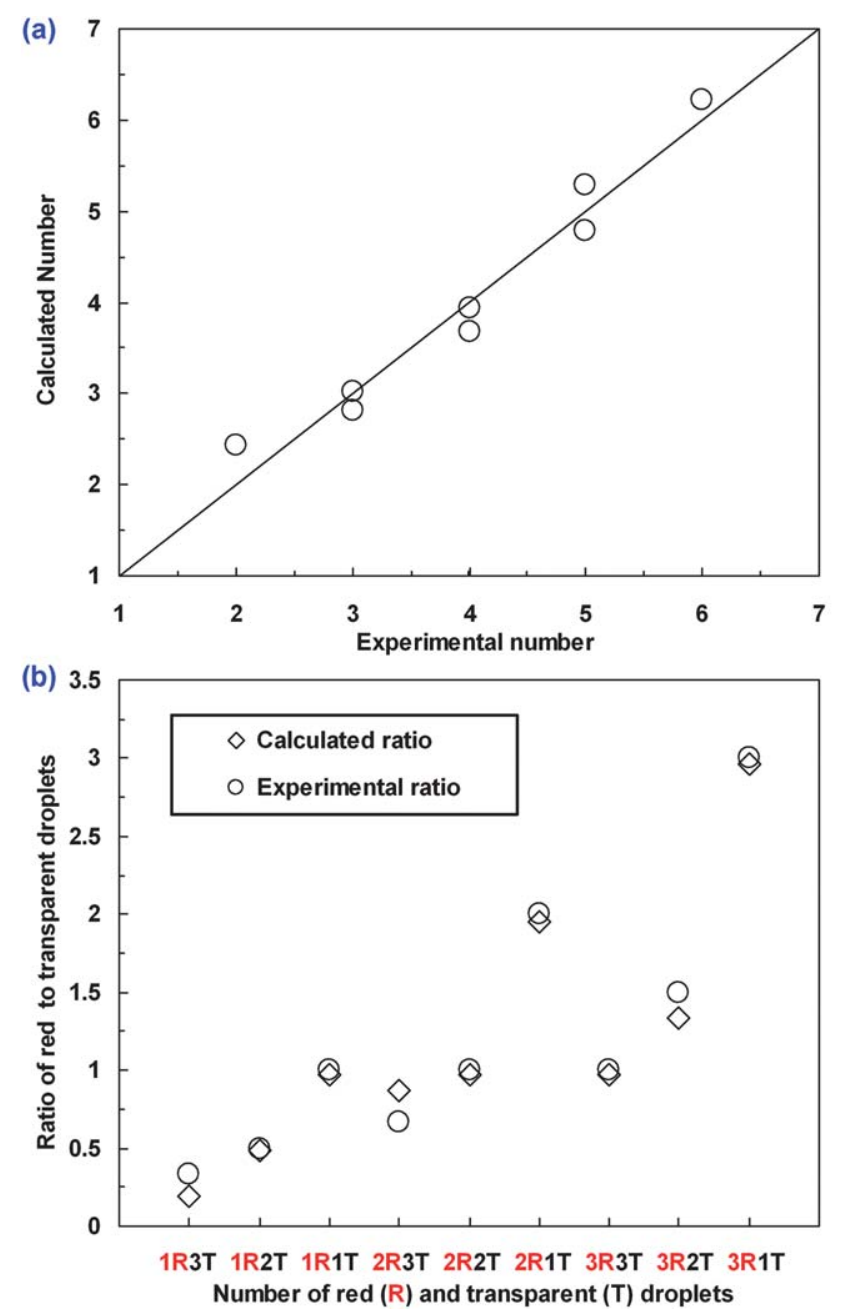

Fig. 3 Prediction for the number $(N)$ (a) and ratio $\left(R_{12}\right)$ (b) of inner red and transparent droplets in quadruple-component double emulsions. (a) Prediction for the value of $N$, where the calculated value of $N$ is obtained from eqn (1). (b) Prediction for the value of $R_{12}$, where the calculated value of $R_{12}$ is obtained from eqn (2). For the device and quadruplecomponent double emulsions shown in Fig. 1, the values of coefficients in eqn (1) and (2) are $a_{1}=0.3957, b_{1}=0.9742, a_{2}=0.2482, b_{2}=1.0137, a_{3}=$ 0.8451 , and $b_{3}=0.4894$.

fluid, further equations can be developed to predict the inner structure of higher-order multicomponent multiple emulsions.

The variety in combinations of the building blocks used in these devices enables simple scale-up for controlled generation of higherorder multicomponent multiple emulsions with more complex structures. This is illustrated by controllably fabricating quintuplecomponent double emulsions, quintuple-component triple emulsions, and even sextuple-component triple emulsions.

For generation of quintuple-component double emulsions, we scale up the microfluidic device in Fig. $1 \mathrm{~b}$ by adding an additional branch channel upstream of the main channel to introduce a third kind of oil droplets into the quadruple-component double emulsions, as shown in Fig. 4a. These additional droplets are dyed with Sudan black, so the quintuple-component $\mathrm{O} / \mathrm{W} / \mathrm{O}$ double emulsions contain oil droplets of three colors. Micrographs of the quintuple-component double emulsions, where the number of each kind of droplets increases from 1 to 5, are shown in Fig. $4 \mathrm{~b}$.

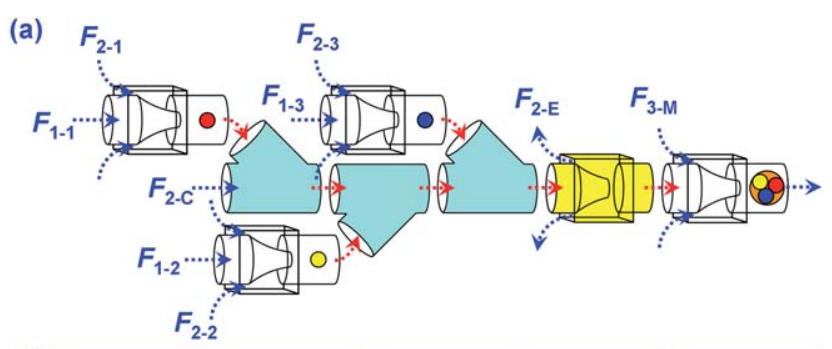

(b)

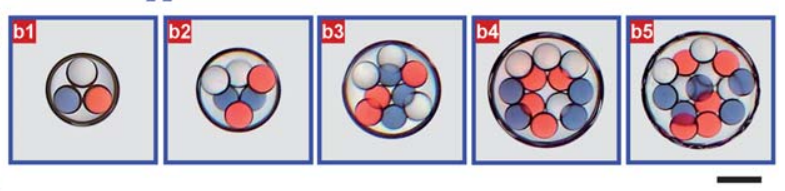

(c)

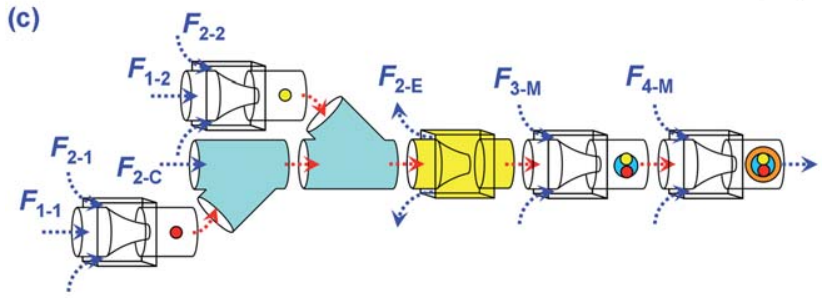

(d) d

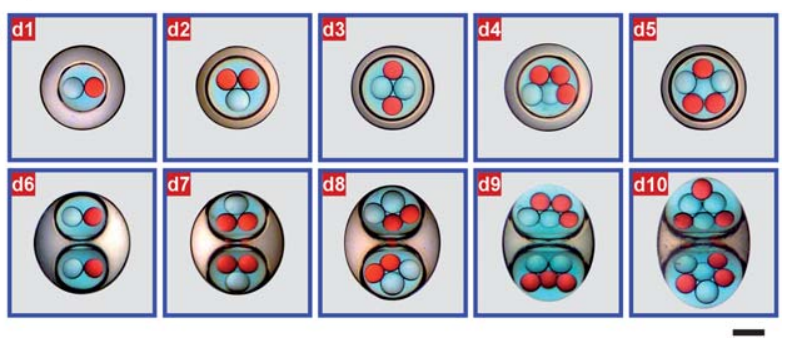

Fig. 4 Extended microfluidic device for controlled production of quintuple-component multiple emulsions. (a) Schematic diagram of the microfluidic device for generating quintuple-component double emulsions. (b) Optical micrographs of quintuple-component double emulsions that contain three different kinds of inner droplets, with a controlled increase in the number of each droplet from 1 to 5. (c) Schematic diagram of the microfluidic device for generating quintuple-component triple emulsions. (d) Optical micrographs of quintuple-component triple emulsions that contain two different kinds of droplets in the innermost level, with precise control over the number and ratio of different innermost droplets and over the number of the middle droplets. Scale bars are $200 \mu \mathrm{m}$.

For fabrication of quintuple-component triple emulsions, an additional drop maker is added downstream in the main channel of the microfluidic device in Fig. 1b to further emulsify the quadruplecomponent double emulsions, as shown in Fig. 4c. The quintuplecomponent oil-in-water-in-oil-in-water $(\mathrm{O} / \mathrm{W} / \mathrm{O} / \mathrm{W})$ triple emulsions are generated with red and transparent oil droplets in the innermost level surrounded by a blue aqueous layer, which is further enveloped in an oil droplet. The number and ratio of innermost red and transparent oil droplets can be precisely controlled as can the number of blue aqueous drops (Fig. 4d).

For generation of more complex sextuple-component triple emulsions, we place two drop makers in each branch channel for the microfluidic device in Fig. $1 \mathrm{~b}$ to generate double emulsions, as shown in Fig. 5a. Because $F_{1-1}, F_{2-1}, F_{1-2}$ and $F_{2-2}$ are each of different components, two kinds of $\mathrm{O} / \mathrm{W} / \mathrm{O}$ double emulsions can be formed in each branch channel, each differing in compositions of both inner and middle phases. After an additional encapsulation of the double 


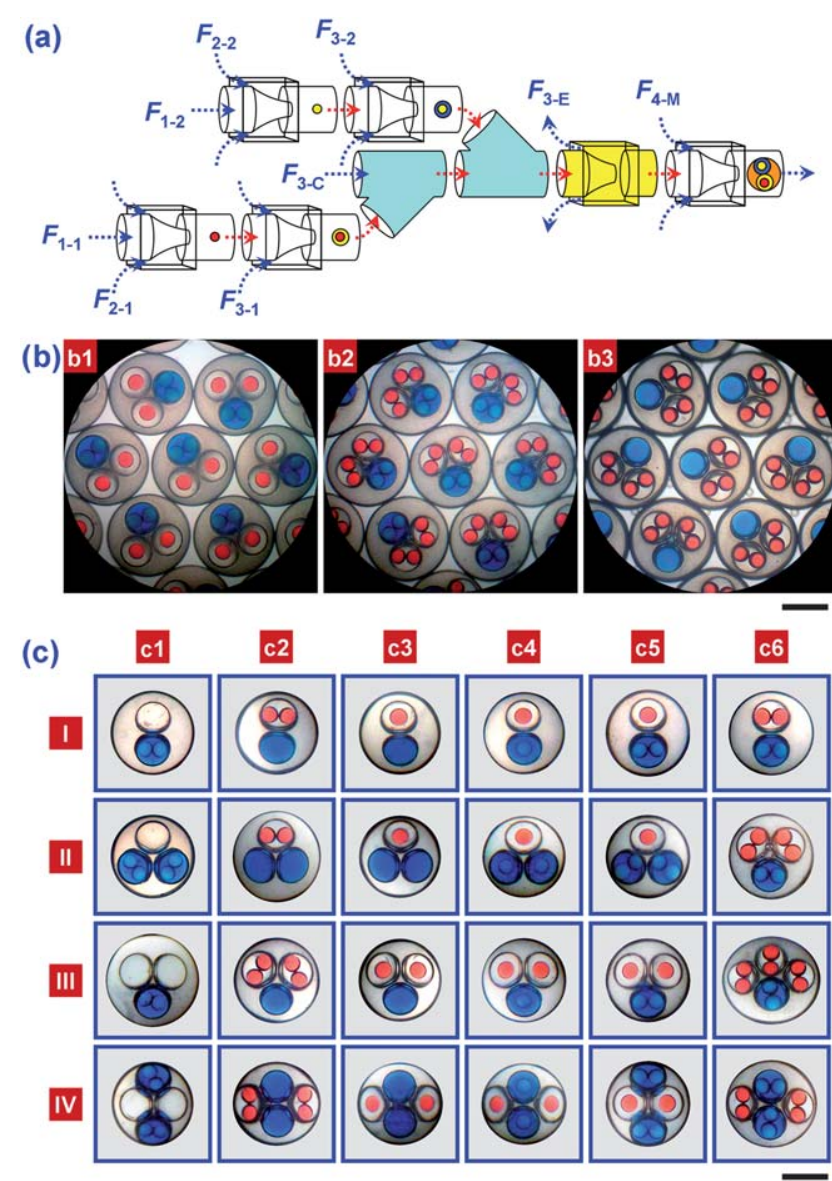

Fig. 5 Extended microfluidic device for controlled production of sextuple-component triple emulsions. (a) Schematic diagram of the microfluidic device for generating sextuple-component triple emulsions. (b) Optical micrographs of monodisperse sextuple-component triple emulsions: (b1 and b2) O/W/O/W triple emulsions containing two different double emulsions and (b3) novel triple emulsions containing one $\mathrm{W} / \mathrm{O}$ single emulsion and two $\mathrm{O} / \mathrm{W} / \mathrm{O}$ double emulsions. (c) Optical micrographs of sextuple-component triple emulsions showing the precise control over the number and ratio of different droplets co-encapsulated in each level of $\mathrm{O} / \mathrm{W} / \mathrm{O} / \mathrm{W}$ triple emulsions. Scale bars are $400 \mu \mathrm{m}$.

emulsions in the main channel, highly controllable and monodisperse sextuple-component triple emulsions are generated, as shown in Fig. 5b. These uniform $\mathrm{O} / \mathrm{W} / \mathrm{O} / \mathrm{W}$ triple emulsions contain two different kinds of double emulsions inside, one of which contains red oil droplets in each transparent aqueous droplet while the other contains transparent oil droplets in each blue aqueous droplet (Fig. 5 (b1) and (b2)). Interestingly, when we stop injecting one of the innermost fluid $\left(F_{1-2}\right)$, a novel type of triple emulsion, which has both double and single emulsions co-encapsulated in the same level, is generated (Fig. 5(b3)). Even with such complex structures, the precise control over the inner structure of sextuple-component triple emulsions is still achievable (Fig. 5c). The number and ratio of the transparent and blue aqueous droplets in the second inner level can be precisely controlled by matching the formation rates of the relevant fluids (Fig. 5c, from row I to row IV). By selectively stopping the injection of either of the innermost fluids, we can produce sextuplecomponent triple emulsions with no red oil droplet in the encapsulated transparent aqueous droplets (Fig. 5c, column c1), or with no transparent oil droplet in the encapsulated blue aqueous droplets (Fig. 5c, columns c2 and c3). We can also achieve precise control over the number of different innermost droplets as well as the ratio of innermost transparent to red droplets (Fig. 5c, columns c4 to c6). All higher-order multicomponent multiple emulsions highlight the remarkable scalability of our microfluidic device; this is crucial because it enables us to arrange the building blocks to engineer multicomponent multiple emulsions with specified complex structures.

The excellent controllability and remarkable scalability of our microfluidic device offer a flexible and valuable route to generating multicomponent multiple emulsions where the number, ratio and size of co-encapsulated multicomponent droplets are each precisely and independently controlled. The co-encapsulated different droplets can function as separate compartments for synergistic delivery of incompatible actives or chemicals, or as microreactor vessels for biochemical or chemical reactions. The high degree of control over the number, ratio and size of different inner droplets enables us to optimize the encapsulation of actives or chemicals and precisely engineer the internals of the multicompartment materials. The approach presented here provides a new method for fabrication and applications of controllable multicomponent multiple emulsions.

\section{Acknowledgements}

The authors gratefully acknowledge support from the NSFC (20825622, 20990220, 20906064, 21036002, 21076127), National Basic Research Program of China (2009CB623407), Specialized Research Fund for Doctoral Program of Higher Education by Ministry of Education of China (200806100038, 20090181120045), the NSF (DMR-1006546) and the Harvard MRSEC (DMR0820484).

\section{References}

1 Z. Nie, S. Xu, M. Seo, P. C. Lewis and E. Kumacheva, J. Am. Chem. Soc., 2005, 127, 8058.

2 S. H. Kim, S. J. Jeon and S. M. Yang, J. Am. Chem. Soc., 2008, 130, 6040.

3 C. H. Chen, A. R. Abate, D. Lee, E. M. Terentjev and D. A. Weitz, Adv. Mater., 2009, 21, 3201.

4 L. Y. Chu, J. W. Kim, R. K. Shah and D. A. Weitz, Adv. Funct. Mater., 2007, 17, 3499.

5 X. Gong, W. Wen and P. Sheng, Langmuir, 2009, 25, 7072.

6 I. G. Loscertales, A. Barrero, I. Guerrero, R. Cortijo, M. Marquez and A. M. Gañán-Calvo, Science, 2002, 295, 1695.

7 S. W. Choi, Y. Zhang and Y. Xia, Adv. Funct. Mater., 2009, 19, 2943.

8 W. Wang, L. Liu, X. J. Ju, D. Zerrouki, R. Xie, L. Yang and L. Y. Chu, ChemPhysChem, 2009, 10, 2405.

9 L. Liu, W. Wang, X. J. Ju, R. Xie and L. Y. Chu, Soft Matter, 2010, 6, 3759.

10 S. Seiffert, J. Thiele, A. R. Abate and D. A. Weitz, J. Am. Chem. Soc., 2010, 132, 6606.

11 H. C. Shum, A. Bandyopadhyay, S. Bose and D. A. Weitz, Chem. Mater., 2009, 21, 5548.

12 R. Engel, S. J. Riggi and M. J. Fahrenbach, Nature, 1968, 219, 856.

13 M. Nakano, Adv. Drug Delivery Rev., 2000, 45, 1.

14 H. Okochi and M. Nakano, Adv. Drug Delivery Rev., 2000, 45, 5.

15 T. Nakashima, M. Shimizu and M. Kukizaki, Adv. Drug Delivery Rev., 2000, 45, 47.

16 S. Higashi and T. Setoguchi, Adv. Drug Delivery Rev., 2000, 45, 57.

17 M. H. Lee, S. G. Oh, S. K. Moon and S. Y. Bae, J. Colloid Interface Sci., 2001, 240, 83.

18 J. Weiss, I. Scherze and G. Muschiolik, Food Hydrocolloids, 2005, 19, 605. 
19 G. Muschiolik, Curr. Opin. Colloid Interface Sci., 2007, 12, 213.

20 B. J. Sun, H. C. Shum, C. Holtze and D. A. Weitz, ACS Appl. Mater. Interfaces, 2010, 2, 3411.

21 H. Chen, Y. Zhao, Y. Song and L. Jiang, J. Am. Chem. Soc., 2008, 130, 7800 .

22 S. Matsumoto, Y. Kita and D. Yonezawa, J. Colloid Interface Sci., 1976, 57, 353.

23 A. S. Utada, E. Lorenceau, D. R. Link, P. D. Kaplan, H. A. Stone and D. A. Weitz, Science, 2005, 308, 537.

24 L. Y. Chu, A. S. Utada, R. K. Shah, J. W. Kim and D. A. Weitz, Angew. Chem., Int. Ed., 2007, 46, 8970.

25 A. R. Abate and D. A. Weitz, Small, 2009, 18, 2030.
26 N. Pannacci, H. Bruus, D. Bartolo, I. Etchart, T. Lockhart, Y. Hennequin, H. Willaime and P. Tabeling, Phys. Rev. Lett., 2008, 101, 164502.

27 W.-A. C. Bauer, M. Fischlechner, C. Abell and W. T. S. Huck, Lab Chip, 2010, 10, 1814.

28 M. Seo, C. Paquet, Z. Nie, S. Xu and E. Kumacheva, Soft Matter, 2007, 3, 986.

29 J. Atencia and D. J. Beebe, Nature, 2005, 437, 648.

30 G. M. Whitesides, Nature, 2006, 442, 368.

31 S. Okushima, T. Nisisako, T. Torii and T. Higuchi, Langmuir, 2004, 20, 9905.

32 T. Nisisako, S. Okushima and T. Torii, Soft Matter, 2005, 1, 23. 